\title{
NOVAS TÉCNICAS AMBULATORIAIS PARA AVALIAÇÃO DA MOTILIDADE ESOFÁGICA E SUA APLICAÇÃO NO ESTUDO DO MEGAESÔFAGO
}

\author{
NEW AMBULATORY TECHNIQUES FOR ASSESSMENT OF ESOPHAGEAL MOTILITY \\ AND THEIR APPLICABILITY ON ACHALASIA STUDY
}

Fernando A. M. Herbella, TCBC-SP' ${ }^{\text {; Jose Carlos Del Grande, TCBC-SP2 }}$

\section{INTRODUÇÃO}

Novos exames ambulatoriais para avaliação da motilidade esofágica tornaram-se acessíveis à prática clinica. Devido a sua introdução relativamente recente, é incerto o real valor desses novos testes no estudo da acalásia.

Trabalhos publicados versando sobre impedanciometria intraluminal multicanal associada à manometria e manometria de alta resolução no estudo da acalásia são brevemente revisados com intuito de estimular futuro estudos, visto que autores nacionais deparam-se rotineiramente com pacientes portadores de megaesôfago, tendo disponibilidade de estudar grande número de pacientes em virtude da Doença de Chagas.

Com este objetivo foi realizada busca na literatura através das bases de dados PubMed, Lilacs e Scielo, sem restrições de linguagem, tipo de estudo ou período de publicação. Foram utilizados os unitermos "acalásia” OU “megaesôfago" E "impedanciometria" OU "manometria de alta resolução".

Foram encontrados seis artigos sobre acalásia e impedanciometria, todos de autores estrangeiros, sendo que todos foram selecionados para análise. Nenhum trabalho foi encontrado sobre manometria de alta resolução e acalasia.

\section{manometria}

Impedanciometria intraluminal multicanal e

A impedanciometria esofágica, criada no início da década de $1990^{1}$, pode ser associada à manometria convencional através de cateter onde sensores de pressão e sensores de impedância são acoplados conjuntamente. A disposição desses sensores é atualmente variável de acordo com o fabricante.

Impedância é a medida em Ohms da resistência elétrica entre dois pontos. Usando sensores pareados em contato com a mucosa do esôfago, o aparelho é capaz de detectar variações na resistência elétrica basal da mucosa esofágica quando substâncias ingeridas (ou refluídas) entram em contato com os sensores e alteram a resistência entre os referidos pontos. Essa tecnologia permite a detecção do bolo alimentar e assim avaliar o seu clareamento e a sua relação com a peristalse (Figura 1).
Devido à diferentes impedâncias, os gases, os líquidos ou a mistura de ambos são facilmente detectados. Os líquidos apresentam baixa resistência, sendo que sua detecção se baseia no decréscimo de $50 \%$ da impedância basal. O oposto é visto com os gases, ou seja, sua detecção baseia-se na elevação da impedância basal em 50\%. A mistura de gases e líquidos mostra uma combinação de ambos. Na deglutição, a variação de impedância é detectada primeiro em sensores proximais e posteriormente nos sensores distais. No caso de refluxo, o oposto é visto. (Figura 2).

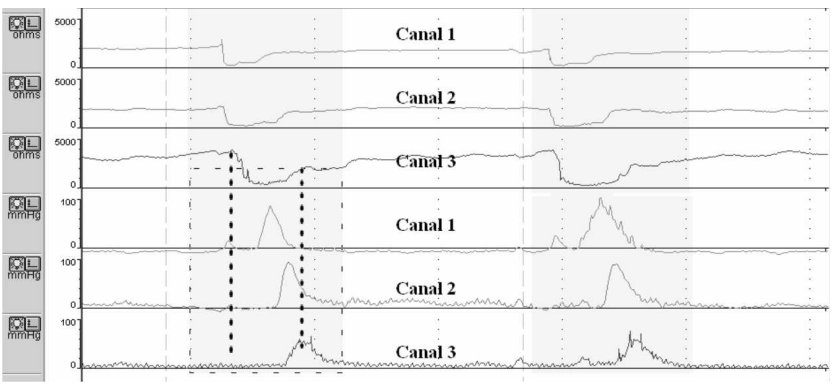

Figura 1 - Traçado normal de impedanciometria mostrando detecção simultânea do trânsito do bolo alimentar (três canais superiores) $e$ da peristalse esofágica (três canais inferiores). Note que o bolo é completamente clareado no momento do pico de contração (linha pontilhada).

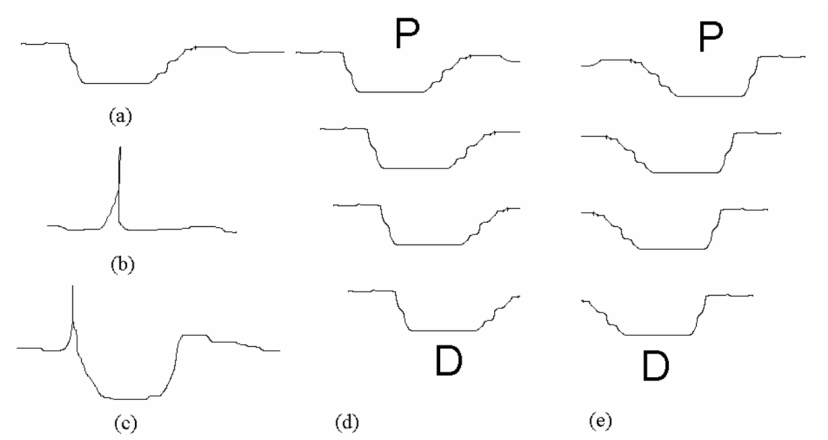

Figura 2 - Traçados de impedanciometria para (a) líquidos; (b) gases; (c) líquidos e gases; (d) deglutição; (e) refluxo. $P$-proximal D-distal

\footnotetext{
1. Médico Assistente do Grupo de Esôfago, Estômago e Intestino Delgado do Departamento de Cirurgia da Universidade Federal de São Paulo - Escola Paulista de Medicina.

2. Professor Adjunto; Chefe do Grupo de Esôfago, Estômago e Intestino Delgado Departamento de Cirurgia da Universidade Federal de São Paulo Escola Paulista de Medicina.
}

Recebido em 27/07/2007

Aceito para publicação em 28/09/2007

Conflito de interesses: nenhum

Fonte de financiamento: nenhuma

Trabalho realizado pelo Grupo de Esôfago, Estômago e Intestino Delgado - Departamento de Cirurgia - Universidade Federal de São Paulo. 
O bolo alimentar é considerado como clareado quando há retorno para $50 \%$ da impedância basal em todos os canais analisados.

Solução salina e não água deve ser usado no exame, com vistas a padronizar a impedância do líquido que será deglutido. Ainda, no exame do corpo esofágico ao invés de apenas 10 deglutições de água (solução salina), 10 deglutições de substância viscosa, yogurte ou geléia, também são utilizadas com propósito de detectar anormalidades sutis da peristalse esofágica. Múltiplos parâmetros podem ser obtidos, contudo, a porcentagem de deglutições nas quais o bolo foi totalmente clareado e o tempo de trânsito são os parâmetros mais usados e suficientemente informativos. Os valores de referência para impedanciometria estão escritos na Tabela 1.

Trabalhos clínicos identificaram quatro padrões de deglutições: (1) ondas peristálticas (baseado na manometria) e bolus clareado (baseado na impedanciometria), (2) ondas peristálticas (baseado na manometria) e bolus não clareado, (3) ondas não peristálticas e bolus clareado (4) ondas não peristálticas e bolus não clareado. A siginificância cliníca desses padrões ainda não foi determinada.

Nos casos de acalásia, devido ao esôfago estar preenchido por líquidos (saliva e restos alimentares não clareados) a impedanciometria mostra valores extremamente baixos de resistência basal ( $<1000$ Ohms) que não se alteram com deglutições (Figura 3) ou ainda, fluxo errático de líquidos em ambos os sentidos (cranial e caudal) (Figura 4). Obviamente, os canais de manometria convencional mostram aperistalse do corpo esofágico com ondas simultâneas e não relaxamento do esfíncter esofagiano inferior.

Diversos estudos demonstraram que em pacientes com acalásia o trânsito do bolo é sempre anormal ${ }^{2-6}$. Nguyen et al. ${ }^{3}$ estudaram 10 pacientes com acalásia que foram comparados à 20 voluntários sadios. Os resultados deste estudo mostraram em pacientes com megaesôfago: (a) menor impedância basal do esôfago, devido ao aumento do calibre do órgão e presença de liquido em seu lúmen; (b) falha no transporte do bolo; (c) regurgitação (peristalse retrograda) e (d) movimento alterado do ar deglutido. Estes autores concluíram que esses parâmetros podem ser usados como padrão para o estudo de outras doenças motoras do esôfago, mas não antevêem aplicabilidade clínica para os achados.

Conchillo et al. ${ }^{7}$ estudaram 10 pacientes com acalásia através de impedanciometria simultânea à videoesofagografia. A detecção do clareamento do bolo alimentar e a altura da coluna de bário foram concordantes em ambos os métodos em pequena porcentagem dos pacientes, sendo bastante dependente do avaliador. Os autores atribuem os resultados à difi- culdades técnicas na interpretação da impedanciometria devido à baixa impedância basal e presença de ar no lúmen do

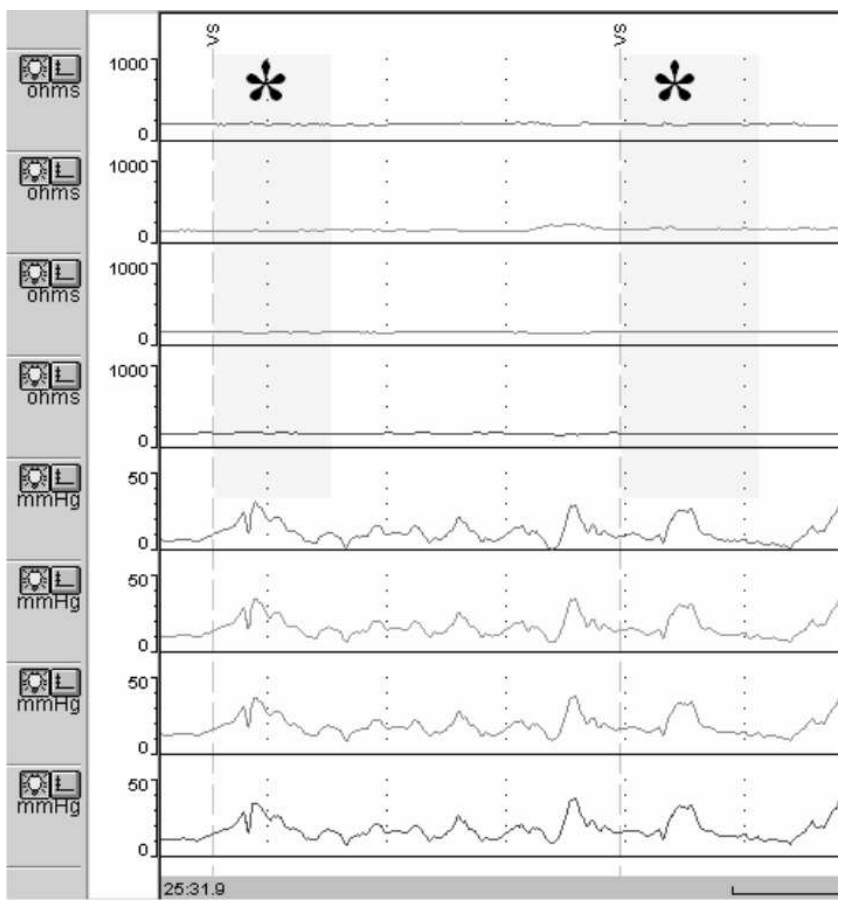

Figura 3 - Traçado de paciente com acalásia na impedanciometria. Valores extremamente baixos de resistência basal ( $<1000$ Ohms) que não se alteram com deglutições são vistos (quatro canais superiores). Manometria convencional mostra aperistalse do corpo esofágico com ondas simultâneas e não relaxamento do esfíncter esofagiano inferior (quatro canais inferiores). * marca deglutições de $5 \mathrm{ml}$ de solução salina.

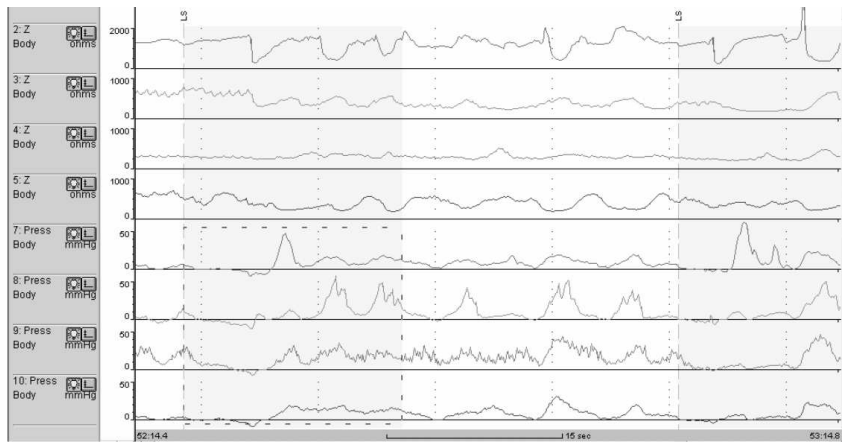

Figura 4 - Traçado de paciente com acalásia na impedanciometria. Impedanciometria mostra fluxo errático de líquidos em ambos os sentidos (cranial e caudal) (quatro canais superiores). Manometria convencional mostra aperistalse do corpo esofágico com ondas simultâneas e não relaxamento do esfíncter esofagiano inferior (quatro canais inferiores).

Tabela 1 - Valores normais para impedanciometria intraluminal multicanal.

\begin{tabular}{llll}
\hline Referencia & Voluntários Estudados & $\begin{array}{c}\text { Clareamento do Bolo } \\
(\% \text { de } 10 \text { deglutições })\end{array}$ & $\begin{array}{c}\text { Tempo de Trânsito } \\
\text { do Bolo }(20 \text { cm })(s)\end{array}$
\end{tabular}

\begin{tabular}{lccccc}
\hline & & Líquidos & Substancias Viscosas & Líquidos & Substancias Viscosas \\
\cline { 3 - 6 } & 42 & 90 & 80 & 14,9 & 17,0 \\
Nguyen et al. & ${ }^{5}$ & 70 & 60 & 11,9 & 12,4 \\
\hline
\end{tabular}


esôfago. Eles concluem que a impedanciometria tem valor limitado na avaliação do esvaziamento esofágico.

\section{Manometria deAlta Resolução}

A manometria de alta resolução é uma variante da manometria convencional na qual múltiplos sensores de pressão estão dispostos no cateter, ao invés de apenas seis a oito. O programa de analise é, assim, capaz de criar um mapa através de contornos isobáricos codificados por cor (Figura 5, esquerda) ou mostrar traçados convencionais em tempo real (Figura 5, direita).

O arranjo dos sensores e variável de acordo com o fabricante. Geralmente, 36 sensores circunferenciais espaçados em $1 \mathrm{~cm}$ é o arranjo mais comum.

É possível com essa tecnologia a aquisição simultânea de dados referentes ao esfíncter esofagiano superior, corpo do esôfago e esfíncter esofagiano inferior. Esse fato reflete em maiores aplicabilidades clínicas e de pesquisa, além de maior conforto ao paciente devido à redução no tempo do exame e sem necessidade de mover o cateter. Os valores de referência mais utilizados estão dispostos na Tabela $2^{8,9}$.

Nos casos de acalásia, ondas simultâneas são facilmente vistas, alem do não relaxamento do esfíncter esofagiano inferior (Figura 6).

A despeito da ainda carência de estudos, a experiência clínica vem demonstrando que a manometria de alta resolução é excelente método para avaliação de defeitos segmentares da peristalse e do relaxamento do esfíncter inferior.

Não há ainda estudos publicados sobre acalásia e manometria de alta resolução.

\section{CONSIDERAÇÕES FINAIS}

Ambas as tecnologias (impedanciometria e manometria de alta resolução) foram introduzidas recentemente na prática clinica. Há ainda escassez de trabalhos clínicos, sendo que apenas dois estudos foram conduzidos especifica- mente em casos de acalásia, nenhum deles enfocando o pósoperatório. Também, ainda não ha publicações de autores brasileiros sobre o assunto.

Esses novos exames certamente permitiram e permitirão uma melhor compreensão da fisiologia da motilidade esofágica, no entanto, ainda é incerto seu valor clinico, especialmente na acalásia.

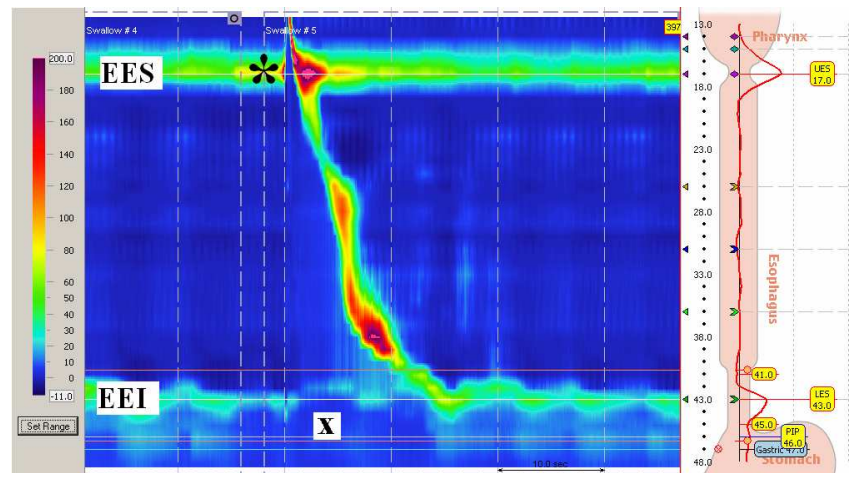

Figura 5 - Traçado normal na manometria de alta resolução. Uma deglutição de $5 \mathrm{ml}$ de água é vista demonstrando o relaxamento oportuno dos esfincteres superior e inferior $(X)$ e propagação da onda peristáltica. EES: esfincter esofagiano superior. EEI: esfincter esofagiano inferior.

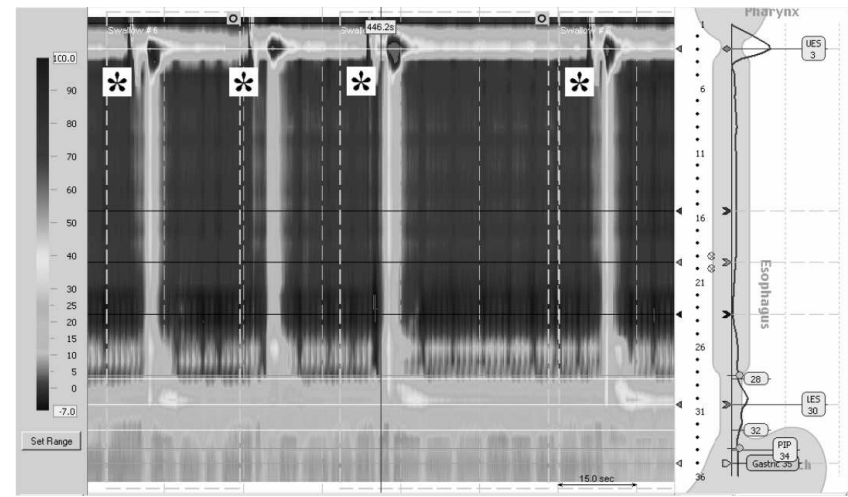

Figura 6 - Traçado de paciente com acalásia na manometria de alta resolução. Ondas simultâneas (*) são vistas, alem do não relaxamento do esfíncter esofagiano inferior.

Tabela 2 - Valores de referência para manometria de alta resolução.

\begin{tabular}{lccccc}
\hline $\begin{array}{l}\text { Pressão } \\
\text { Basal doEEI }\end{array}$ & $\begin{array}{c}\text { Pressão Residual } \\
\text { doEEI }\end{array}$ & $\begin{array}{c}\text { Amplitudeno } \\
\text { Esôfago Proximal }\end{array}$ & $\begin{array}{c}\text { Duração da Contração } \\
\text { no Esôfago Proximal }\end{array}$ & $\begin{array}{c}\text { Amplitude no } \\
\text { Esôfago Distal }\end{array}$ & $\begin{array}{c}\text { Duração da Contra- } \\
\text { ção no Esôfago Distal }\end{array}$ \\
\hline $5,0-31,6$ & $0-10,1$ & $53,7-185,3$ & $1,2-4,8$ & $47,2-216,8$ & $0,6-6,6$ \\
\hline
\end{tabular}

EEI-esfincter esofagiano inferior.

Pressões em $\mathrm{mmHg}$.

\begin{abstract}
Brazilian surgeons deal routinely with esophageal motility disorders, because achalasia is highly prevalent in Brazil due to Chagas' disease. In the last years new technologies for the evaluation of esophageal motility became available. High resolution manometry and the combination of barometric parameters and intraluminal impedance are the new frontiers on this topic. The authors reviewed current, national and international, literature about achalasia with multichannel intraluminal impedance and high resolution manometry studies. The new technologies described are promising, however few studies have been published and further studies are still expected for achalasia patients (Rev. Col. Bras. Cir. 2008; 35(3): 199-202).
\end{abstract}

Key words: Esophageal motility disorders; Achalasia; Manometry; Impedance. 


\section{REFERENCIAS}

1. Silny J. Intraluminal multiple electric impedance procedure for measurement of gastrointestinal motility. J Gastrointest Mot. 1991; 3:151-62.

2. Conchillo JM, Nguyen NQ, Samsom M, Holloway RH, Smout AJ. Multichannel intraluminal impedance monitoring in the evaluation of patients with non-obstructive dysphagia. Am J Gastroenterol. 2005; 100(12):2624-32.

3. Nguyen HN, Domingues GR, Winograd R, Lammert F, Silny J, Matern S. Impedance characteristics of esophageal motor function in achalasia. Dis Esophagus. 2004; 17(1):44-50.

4. Tutuian R, Castell DO. Combined multichannel intraluminal impedance and manometry clarifies esophageal function abnormalities: study in 350 patients. Am J Gastroenterol. 2004; 99(6):1011-9.

5. Nguyen NQ, Rigda R, Tippett M, Conchillo J, Smout AJ, Holloway RH. Assessment of oesophageal motor function using combined perfusion manometry and multi-channel intra-luminal impedance measurement in normal subjects. Neurogastroenterol Motil. 2005; 17(3):458-65.

6. Tutuian R, Vela MF, Balaji NS, Wise JL, Murray JA, Peters JH, Shay SS, Castell DO. Esophageal function testing with combined multichannel intraluminal impedance and manometry: multicenter study in healthy volunteers. Clin Gastroenterol Hepatol. 2003; 1(3):174-82.
7. Conchillo JM, Selimah M, Bredenoord AJ, Samsom M, Smout AJPM. Assessment of oesophageal emptying in achalasia patients by intraluminal impedance monitoring. Neurogastroenterol Motil. 2006; 18(11):971-7.

8. Pandolfino JE, Ghosh SK, Zhang Q, Jarosz A, Shah N, Kahrilas PJ. Quantifying EGJ morphology and relaxation with highresolution manometry: a study of 75 asymptomatic volunteers. Am J Physiol Gastrointest Liver Physiol. 2006; 290(5):G103340. Epub 2006 Feb 2.

9. Ghosh SK, Pandolfino JE, Zhang Q, Jarosz A, Shah N, Kahrilas PJ. Quantifying esophageal peristalsis with high-resolution manometry: a study of 75 asymptomatic volunteers. Am J Physiol Gastrointest Liver Physiol. 2006; 290(5):G988-97. Epub 2006 Jan 12.

Como citar este artigo:

Herbella FAM, Del Grande JC. Novas técnicas ambulatoriais para avaliação da motilidade esofágica e sua aplicação no estudo do megaesôfago. Rev Col Bras Cir. [periódico na Internet] 2008; 35(3). Disponível em URL: http://www.scielo.br/rcbc

Endereço para correspondência:

Fernando Herbella

601 Elmwood Ave, Box Surg

Rochester, NY, USA, 14642

Tel:+1(585)276-3194

herbella.dcir@unifesp.epm.br 